\title{
Control of light polarization by voltage in excitonic metasurface devices
}

Cite as: Appl. Phys. Lett. 111, 241101 (2017); https://doi.org/10.1063/1.5005827

Submitted: 19 September 2017 . Accepted: 22 November 2017 . Published Online: 11 December 2017

S. V. Lobanov, N. A. Gippius, S. G. Tikhodeev (D), and L. V. Butov
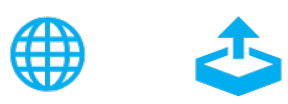

\section{ARTICLES YOU MAY BE INTERESTED IN}

High-efficiency and low-loss gallium nitride dielectric metasurfaces for nanophotonics at visible wavelengths

Applied Physics Letters 111, 221101 (2017); https://doi.org/10.1063/1.5007007

Broadband cross-polarization conversion by symmetry-breaking ultrathin metasurfaces Applied Physics Letters 111, 241108 (2017); https://doi.org/10.1063/1.5006540

Dual-surface flexible THz Fano metasensor

Applied Physics Letters 111, 201101 (2017); https://doi.org/10.1063/1.5000428

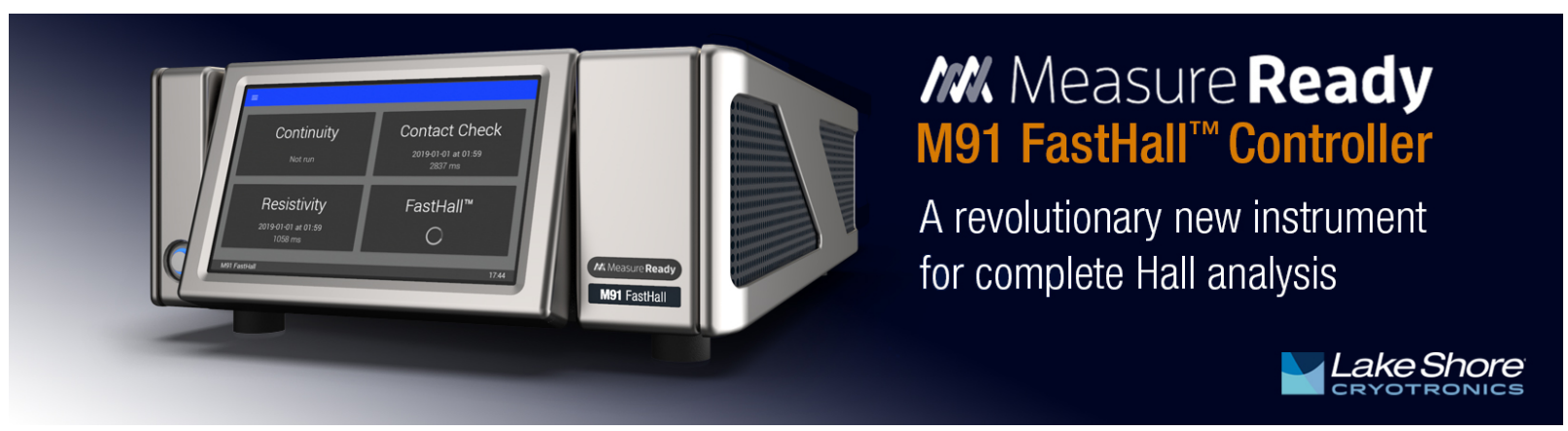




\title{
Control of light polarization by voltage in excitonic metasurface devices
}

\author{
S. V. Lobanov, ${ }^{1,2}$ N. A. Gippius, ${ }^{2}$ S. G. Tikhodeev, ${ }^{3,4}$ and L. V. Butov ${ }^{5}$ \\ ${ }^{1}$ School of Physics and Astronomy, Cardiff University, Cardiff CF24 3AA, United Kingdom \\ ${ }^{2}$ Skolkovo Institute of Science and Technology, Moscow 143026, Russia \\ ${ }^{3}$ A. M. Prokhorov General Physics Institute, Russian Academy of Sciences, Vavilova Street 38, Moscow \\ 119991, Russia \\ ${ }^{4}$ M. V. Lomonosov Moscow State University, Leninskie Gory 1, Moscow 119991, Russia \\ ${ }^{5}$ Department of Physics, University of California at San Diego, La Jolla, California 92093-0319, USA
}

(Received 19 September 2017; accepted 22 November 2017; published online 11 December 2017)

\begin{abstract}
We propose active metasurface devices where the state of emitted light is controlled by voltage. Based on the simulations of expected emission spectra, we present the concept of a light emitting device with the voltage controlled wavelength and degree of linear polarization of emission. The device combines the ability of metasurfaces to control light with a wavelength-tunable light source based on indirect excitons in coupled quantum well heterostructures. (C) 2017 Author(s). All article content, except where otherwise noted, is licensed under a Creative Commons Attribution (CC BY) license (http://creativecommons.org/licenses/by/4.0/). https://doi.org/10.1063/1.5005827
\end{abstract}

Plasmonic metasurfaces exploring plasmonic resonances in a metal grating on a dielectric substrate are now widely used for various purposes such as to enhance the efficiency of light emitting devices (LEDs), ${ }^{1}$ to magnify the light absorption in photodetectors ${ }^{2}$ and thin film solar cells, ${ }^{3,4}$ to control the intensity and directivity of light emission (as nanoantennas), ${ }^{5}$ to enhance the magneto-optical effects ${ }^{6-8}$ and the sensitivity of optical sensors for chemical gas detection and biosensing, ${ }^{9-11}$ to magnify nonlinear optical effects such as 2nd and 3rd harmonic generation, ${ }^{12}$ and in photochemistry. ${ }^{13}$

In this letter, we propose to combine the ability of plasmonic metasurfaces to control light with a wavelengthtunable light source based on indirect excitons (IXs) for creating integrated optoelectronic devices where the polarization state of emitted light is controlled by voltage. IXs are composed of electrons and holes in spatially separated quantum well layers [Fig. 1(b)]. Due to the IX built-in electric dipole moment $e d$, the energy of photons emitted by IXs is effectively controlled by voltage $\delta E=-e d F_{z}$, where $d$ is the separation between the electron and hole layers (for coupled quantum wells, CQWs, $d$ is close to the distance between the QW centers) and $F_{z} \propto V$ is an electric field perpendicular to the QW plane. The IX energy control by voltage allowed creating a variety of excitonic devices, including traps and lattices for studying basic phenomena in IXs, as well as excitonic transistors, routers, and photon storage devices, which form the potential for creating excitonic signal processing devices and excitonic circuits. ${ }^{1-16}$

Specifically, in this letter, based on the theoretical analysis of simulated emission spectra, we put forward a concept of tunable excitonic metasurface devices. Namely, we propose a light emitting device with the voltage controlled wavelength and degree of linear polarization (DLP) of emission made of plasmonic metal grating deposited on top of an AlGaAs/GaAs CQW structure. The metal grating is dualpurpose. On the one hand, it serves as the upper electrode to control the IX emission wavelength. On the other hand, it provides plasmonic resonances, allowing us to control the DLP of IX photoluminescence.
The schematic diagram of the proposed device is shown in Fig. 1(a). CQW (black dashed lines) is positioned within an undoped $1 \mu \mathrm{m}$ thick $\mathrm{Al}_{0.33} \mathrm{Ga}_{0.67}$ As layer between a
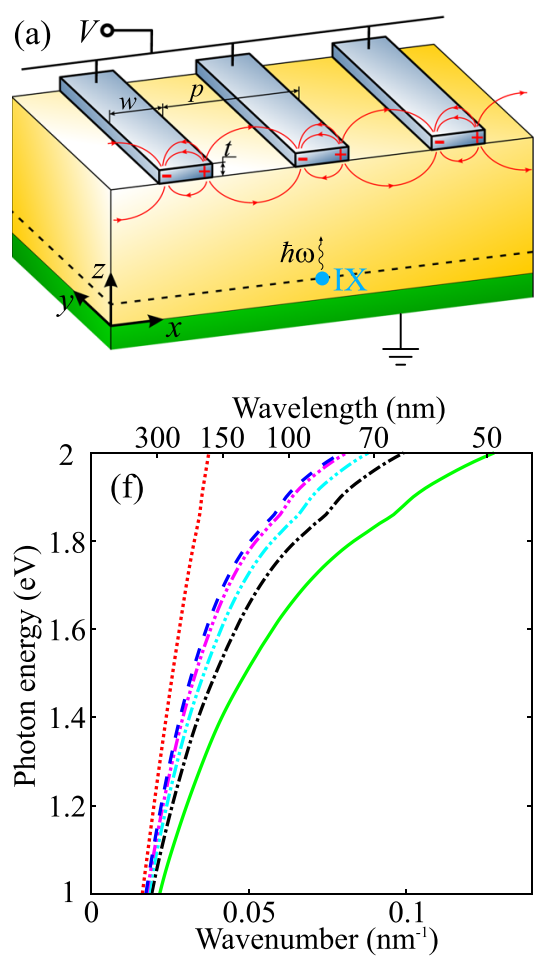

(b)
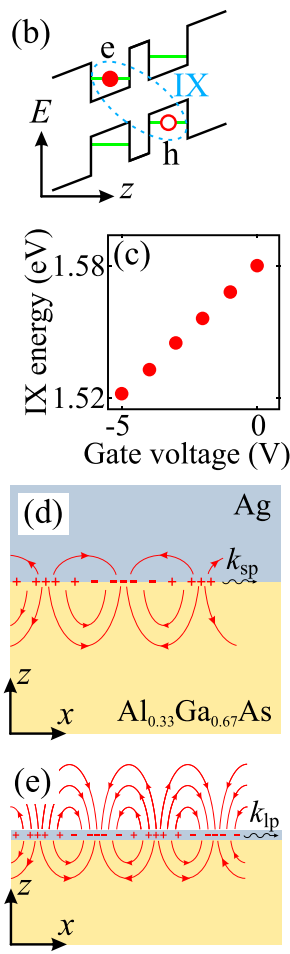

FIG. 1. (a) Schematic diagram of the device. Coupled quantum well, CQW (black dashed lines), is positioned within an undoped $1 \mu \mathrm{m}$ thick $\mathrm{Al}_{0.33} \mathrm{Ga}_{0.67} \mathrm{As}$ layer (yellow) between a conducting $n^{+}$-GaAs layer serving as a homogeneous bottom electrode (green) and a striped top electrode (silvery). (b) Energy band diagram of the CQW; e, electron; h, hole. The cyan dashed ellipse indicates an indirect exciton (IX). (c) Energy of IX as a function of applied voltage. The data are taken from Ref. 14. (d) and (e) Schematic of SPP propagation along a metal-dielectric boundary (d) and a thin metal film in asymmetric dielectric surrounding (e). (f) Calculated dispersions of $\mathrm{Ag} / \mathrm{AlGaAs}$ SPP (blue dashed line) and air/Ag/AlGaAs thinlayer SPP for the silver film with thickness $t=10 \mathrm{~nm}$ (green solid line), $15 \mathrm{~nm}$ (black dashed-dotted line), $20 \mathrm{~nm}$ (cyan dashed-double-dotted line), and $30 \mathrm{~nm}$ (magenta dashed-triple-dotted line). The red dotted line shows the light cone in $\mathrm{Al}_{0.33} \mathrm{Ga}_{0.67} \mathrm{As}$. 
conducting $n^{+}$-GaAs layer serving as a homogeneous bottom electrode and a top electrode made of Ag nanowire grating. The nanowires have thickness $t$, width $w$, and grating period $p$.

The IX photoluminescence from CQW plays the role of a monochromatic source of unpolarized light with the voltage-tunable wavelength. The source of IXs can be, e.g., an optical pump in some neighboring part of the device, as it was done in Refs. 14 and 16. The CQW band diagram is shown in Fig. 1(b); a typical dependence of the IX energy $E(V)$ on the applied voltage is shown in Fig. 1(c).${ }^{14}$ Note however that $E(V)$ can be optimized for a tunable metasurface device by adjusting the CQW parameters including the QW widths, width of the barrier between the QWs, and the well and barrier materials. Figure 1(c) shows that changing the applied voltage allows us to scan the energy of IX photoluminescence in the range of $1520-1580 \mathrm{meV}$. If the metal grating is engineered in such a way that one of its plasmon resonances is in the same energy range, it becomes possible to change the DLP of the device emission, due to pronounced changes of $x$-polarized light transmission of the grating in the vicinity of the plasmonic resonances.

The characteristic energy dispersions of plasmons in metal gratings can be simply understood as follows. We start from a continuous metal film. Figure 1(d) shows schematically the electric field of a surface plasmon (SP) polariton (SPP), propagating along a boundary between a dielectric and a semi-infinite metal. The calculated SPP energy dispersion as a function of wavenumber $k_{\mathrm{sp}}=2 \pi / \lambda_{\mathrm{sp}}$ (where $\lambda_{\mathrm{sp}}$ is the SPP wavelength) is shown for the $\mathrm{Ag} / \mathrm{Al}_{0.33} \mathrm{Ga}_{0.67} \mathrm{As}$ boundary by the blue dashed line in Fig. 1(f). It is located below the $\mathrm{Al}_{0.33} \mathrm{Ga}_{0.67} \mathrm{As}$ light cone [red dotted line in Fig. 1(f)]. The SPP dispersion curve for the air/Ag boundary (not shown) lies below the air light cone (also not shown); both are significantly blue shifted due to a large refraction index of semiconducting AlGaAs.

If a semi-infinite metal is replaced by a metal film of finite thickness in asymmetric dielectric surrounding (air and $\mathrm{Al}_{0.33} \mathrm{Ga}_{0.67} \mathrm{As}$ in our case), the plasmons propagating on the opposite sides of the metal film interact with each other. The lower-energy SPP (localized predominantly on the AlGaAs side of the film) repulses from the upper-energy SPP (localized predominantly on the air side). Resultantly, this yields a red energy shift of the lower-energy SPP with the decrease in the film thickness. The calculated dispersions of the lower-energy SPP for the silver film with thickness $t=10 \mathrm{~nm}$ (green solid line), $15 \mathrm{~nm}$ (black dashed-dotted line), $20 \mathrm{~nm}$ (cyan dasheddouble-dotted line), and $30 \mathrm{~nm}$ (magenta dashed-triple-dotted line) are shown in Fig. 1(f). The dispersions are calculated neglecting absorption loses in silver and $\mathrm{Al}_{0.33} \mathrm{Ga}_{0.67} \mathrm{As}$.

Note that the polarization of all such SPPs is always magnetic field $\mathbf{H} \| y$ [see in Fig. 1(a)], i.e., along the boundaries and perpendicular to the SPP propagation direction $x$. As to the SSP electric field [shown schematically in Figs. 1(d) and 1(e)], there are both transverse and longitudinal components, $E_{z}$ and $E_{x}$, respectively.

If the metal film is truncated from both sides into a single metal nanowire of width $w$, the localized Mie plasmons arise due to SPP reflections back and forth, forming standing waves. Formation of such localized plasmons requires the width of the wire to be roughly an integer number of thinlayer plasmon half wavelength, i.e.,

$$
w_{m}=m \lambda_{\mathrm{lp}} / 2 \text {, }
$$

where $\lambda_{\mathrm{lp}}$ is the wavelength of the thin-layer plasmon and $m$ is a positive integer. Note that Eq. (1) assumes quasi onedimensional geometry and perfect reflection at the nanowire boundaries and thus provides only qualitative matching between $w$ and $\lambda_{\text {lp }}$. One can see from Fig. 1(f) that, e.g., the 10 -nm-thick-layer plasmon wavelength is $\lambda_{\mathrm{lp}} \approx 120 \mathrm{~nm}$ for the IX emission energy $\hbar \omega=1.55 \mathrm{eV}$.

The calculated dependence of the $x$-linear polarized component of the IX device emission efficiency $I_{x}$ along the $z$-axis on the nanowire width $w$ and period $p$ for the photon energy $\hbar \omega=1.55 \mathrm{eV}$ and thickness $t=10 \mathrm{~nm}$ is shown in Fig. 2(a). In this and other figures, the emission efficiency is calculated as the emission intensity normalized to the maximum emission intensity of an equivalent IX in a homogeneous semiconductor $\mathrm{Al}_{0.33} \mathrm{Ga}_{0.67} \mathrm{As}$. We assume that IXs are uniformly distributed over the CQW and radiate light incoherently with random polarization. In order to calculate the emission intensity, we consider the weak coupling limit, employ the optical scattering matrix method ${ }^{17,18}$ and the electrodynamical reciprocity principle as described in Refs.19-21, and use the dielectric susceptibility of $\mathrm{Ag}$ from Ref. 22 and $\mathrm{Al}_{0.33} \mathrm{Ga}_{0.67} \mathrm{As}$ from Ref. 23. The emission is calculated in the direction normal to the device [along the $z$-axis, see in Fig. 1(a)]. The calculated directional diagrams for devices with parameters displayed in Figs. 2(e), 2(f), and 3(b) are given in the supplementary material for completeness.

Three resonance branches are clearly seen in Fig. 2(a) that correspond to excitation of localized SPPs. These SPPs weakly depend on the period $p$. In a good qualitative agreement with Eq. (1) for odd $m$, these SPPs occur at nanowire widths $w_{1} \approx 40 \mathrm{~nm} \approx \lambda_{\mathrm{lp}} / 2, w_{3} \approx 150 \mathrm{~nm} \approx 3 \lambda_{\mathrm{lp}} / 2, \quad$ and $w_{5} \approx$ $270 \mathrm{~nm} \approx 5 \lambda_{\mathrm{lp}} / 2$. The respective DLP $\rho=\left(I_{x}-I_{y}\right) /\left(I_{x}+I_{y}\right)$ is shown in Fig. 2(b). Here, $I_{y}$ is the $y$-linear polarized component of the IX device emission efficiency. One can see that DLP is varied in the range from about $0 \%$ (unpolarized emission) to about $-99 \%$ for the first plasmon branch $(w \approx 40 \mathrm{~nm})$ and to about $-85 \%$ for the second branch $(w \approx 150 \mathrm{~nm})$.

Calculated $x$ - (blue solid line) and $y$ - (green dashed line) linear polarized components of the IX device emission spectra and respective DLP $\rho$ (red dotted line) for the structures with the nanowire thickness $t$, width $w$, and period $p$, where $(t, w, p)=(10,40,100) \mathrm{nm}$ and $(10,150,210) \mathrm{nm}$, are shown in Figs. 2(c) and 2(d), respectively.

In order to understand the effectiveness of the excitation of localized SPPs, we show in Figs. 2(e) and 2(f) the calculated electric field distribution of the light propagating along the $z$-axis with photon energies of $1531 \mathrm{meV}$ and $1563 \mathrm{meV}$, for structures in Figs. 2(c) and 2(d), respectively. The distributions have one and three anti-nodes, respectively, in agreement with the above discussion. Note that SPPs with even $m$ have an even distribution of the electric field with respect to reflection in the vertical symmetry plane of the structure and consequently cannot emit light along the $z$-axis for the structure of interest with spatially uniform excitation of CQW.

In the above described cases of thin metal wires, the transmissivity of the grating in both linear polarisations along 

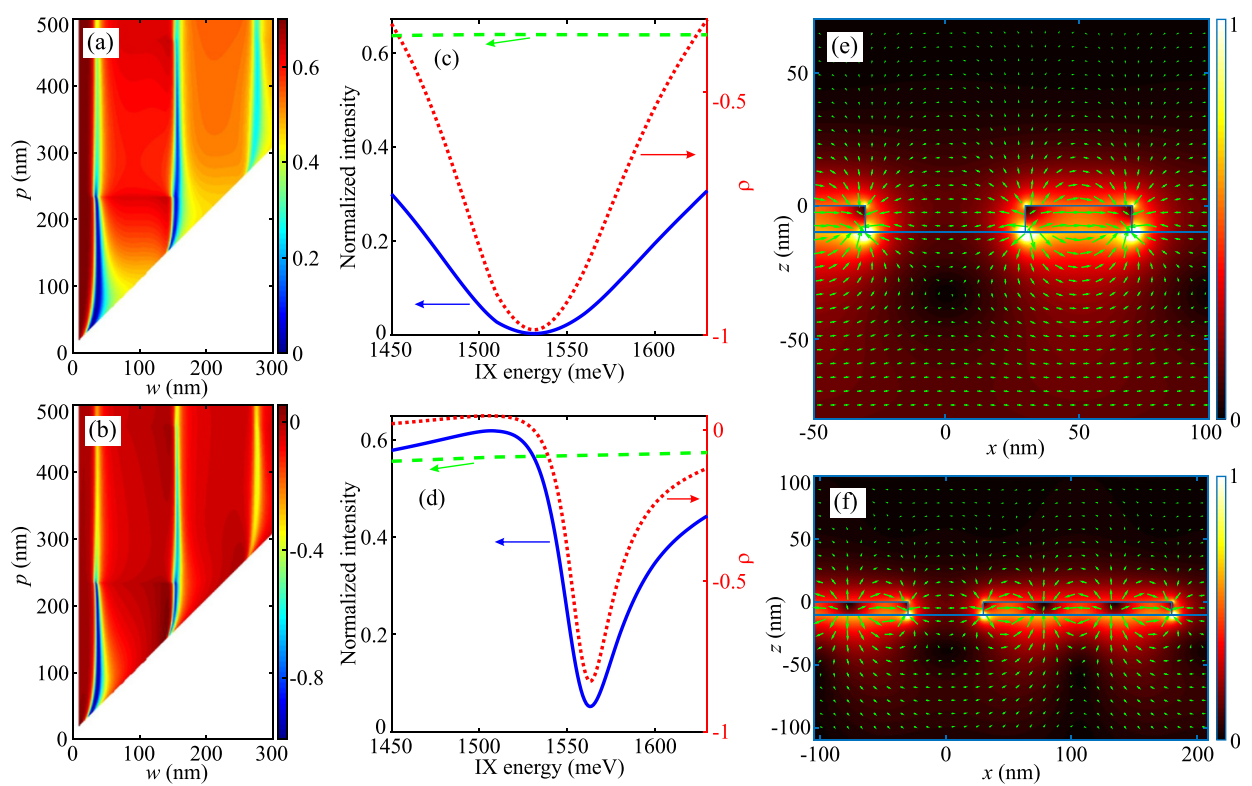

FIG. 2. (a) Calculated dependence of the $x$-linear polarized component of the IX device emission efficiency $I_{x}$ along the $z$-axis on the nanowire width $w$ and period $p$ for the photon energy $\hbar \omega=1.55 \mathrm{eV}$ and nanowire thickness $t=10 \mathrm{~nm}$. (b) Respective degree of linear polarization $\rho$. (c) and (d) Calculated $x$ - (blue solid line) and $y$ - (green dashed line) linear polarized components of the emission spectra and respective degree of linear polarization $\rho$ (red dotted line) for the structures with the stripe thickness $t$, width $w$, and period $p$, where (c) $(t, w, p)=(10,40,100) \mathrm{nm}$ and (d) $(10,150,210) \mathrm{nm}$, shown as functions of voltage-controlled IX emission energy. (e) and (f) Calculated electric field distribution of the light propagating along the $z$-axis with IX emission energy of (e) $\hbar \omega=1531 \mathrm{meV}$ and (f) $1563 \mathrm{meV}$ for the structures with (e) $(t, w, p)=(10,40,100) \mathrm{nm}$ and (f) $(10,150,210) \mathrm{nm}$. The background color shows the absolute value of the electric field.

and perpendicular to the wires is relatively high away from the SPP resonances. The emission of our device becomes strongly linearly polarized along the wires $(\| y, \rho \sim-1)$ at the SPP resonance frequency, because the grating becomes nearly opaque for $x$-polarized light.

Using thick metal gratings and the famous effect of the extraordinary optical transmission (EOT) through arrays of subwavelength holes ${ }^{24-27}$ it is also possible to receive nearly completely $x$-polarized light emission of IXs near the SPP resonance frequency.
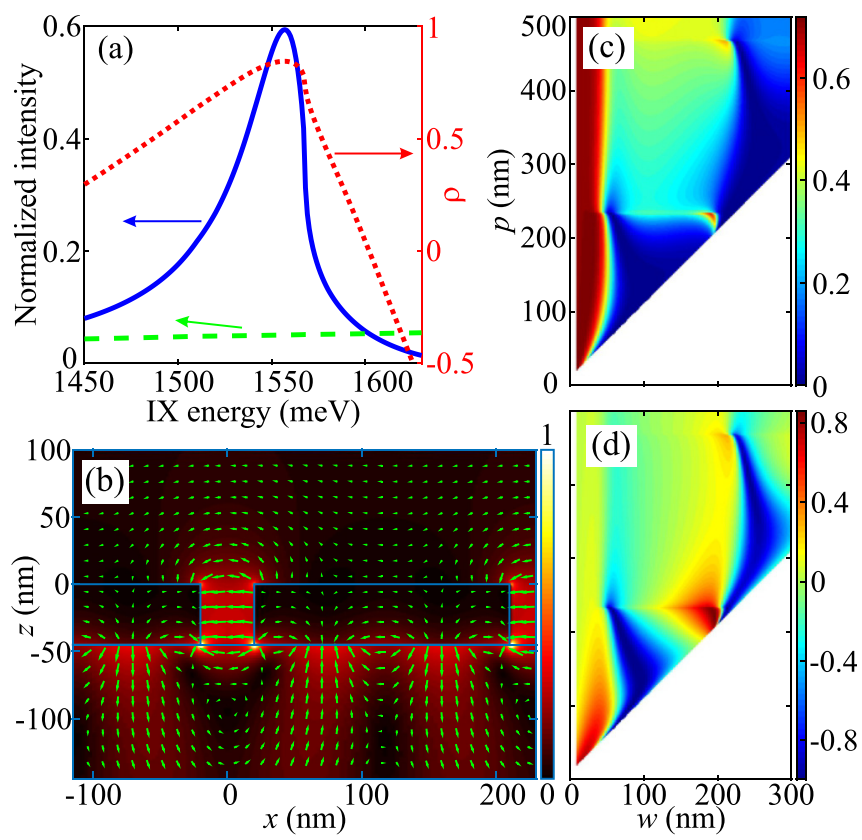

FIG. 3. (a) As Fig. 2(c), but for $(t, w, p)=(45,190,230) \mathrm{nm}$. (b) As Fig. 2(e), but for $\hbar \omega=1557 \mathrm{meV}$ and $(t, w, p)=(45,190,230) \mathrm{nm}$. (c) and (d) As Figs. 2(a) and 2(b), but for $t=45 \mathrm{~nm}$.
If the thickness of a homogeneous metal film is larger than the skin depth of the metal, which is about $20 \mathrm{~nm}$ for silver at $\hbar \omega=1.55 \mathrm{eV}$, the interaction of surface plasmons propagating along opposite sides of the film is weak. Therefore, one can consider them independently and use the surface-plasmon dispersion curve [blue dashed line in Fig. 1(f)] for analytical estimations. One can see from Fig. 1(f) that the wavelength of the surface plasmon for photon energy $\hbar \omega=1.55 \mathrm{eV}$ is $\lambda_{\text {sp }}=184 \mathrm{~nm}$.

If one makes narrow slits in a thick silver film, light can transmit through them. The narrower the slits are the smaller the transmission is. However, if the slits are ordered in a periodic array with period $p$, both light propagating along the $z$-axis in $\mathrm{Al}_{0.33} \mathrm{Ga}_{0.67} \mathrm{As}$ and surface plasmon propagating along the $x$ axis along the $\mathrm{Ag}-\mathrm{Al}_{0.33} \mathrm{Ga}_{0.67} \mathrm{As}$ boundary undergo Bragg diffraction and interact with electromagnetic waves, the in-plane wavenumber of which differs by $2 \pi m / p$, where $m$ is an integer. Taking into account that light propagating along the $z$-axis has zero in-plane wavenumber, one conclude that this light can excite surface plasmons with wavelength $\lambda_{\mathrm{sp}}$ if the period of the grating is $p=m \lambda_{\mathrm{sp}}$, where $m$ is a positive integer. The surface plasmon in turn enhances the electric field in the hole region, thus yielding EOT through the metal film with slits.

The calculated $x$ - (blue solid line) and $y$ - (green dashed line) linear polarized components of the IX device emission spectra and respective DLP $\rho$ (red dotted line) for the structure with $(t, w, p)=(45,190,230) \mathrm{nm}$ are shown in Fig. $3(\mathrm{a})$. One can see a narrow resonance with extraordinary emission of $x$-polarized light here. Note that since the wavelengths of surface plasmon and light in $\mathrm{Al}_{0.33} \mathrm{Ga}_{0.67} \mathrm{As}$ are about the same for the photon energy of $1.55 \mathrm{eV}$ [compare blue dashed and red dotted lines in Fig. 1(f)], the $x$-linear polarized component of the IX's emission decreases rapidly from the right side of the resonance [blue line in Fig. 3(a)] 
due to opening new diffraction channels in AlGaAs (a Wood-Rayleigh anomaly).

The calculated electric field distribution of light propagating along the $z$-axis with photon energy equal to the resonance energy (1557 meV) is shown in Fig. 3(b). One can clearly see the excitation of SPP propagating along the $\mathrm{Ag}$ / $\mathrm{Al}_{0.33} \mathrm{Ga}_{0.67} \mathrm{As}$ boundary and enhancement of the electric field in the hole region as it was discussed above. Note also that the surface plasmon forms a standing wave or, in other words, propagates in both directions due to mirror symmetry of the considered structure and normal light incidence.

The calculated dependence of the $x$-linear polarized component of the IX's emission efficiency $I_{x}$ along the $z$-axis on the nanowire width $w$ and period $p$ for the photon energy $\hbar \omega=1.55 \mathrm{eV}$ and stripe thickness $t=45 \mathrm{~nm}$ is shown in Fig. 3(c). A narrow resonance with extraordinary emission of $x$-polarized light occurs around the nanowire width $w=190 \mathrm{~nm}$ and period $p=230 \mathrm{~nm}$. These parameters correspond to the case of the small slit between neighbouring nanowires $g=40 \mathrm{~nm}$. Respective DLP $\rho$ is shown in Fig. $3(d)$. One can see that DLP is varied in the range from about $-100 \%$ to about $+85 \%$.

We also compared the device performance for various electrode materials. The estimates in the supplementary material show linear polarized components of the IX device emission spectra and respective DLP for voltage tunable excitonic metasurface devices with gold gratings (Figs. S4-S6, supplementary material) operating in the same modes [see Figs. 2(c), 2(d), and 3(a)] as the light emission device with silver gratings. The DLP for these structures is varied in the smaller range than for the silver ones because of larger absorptive losses of gold in the investigated frequency range. Smaller DLP variations are estimated for metal nitride plasmonic materials ${ }^{28}$ with larger losses such as titanium nitride, see Fig. S7 (supplementary material).

Compared to a more traditional way to control the light polarization employing liquid crystals, ${ }^{29}$ the wavelength of IX emission (and thus the polarization of emission in our device) can be changed much faster, on characteristic times down to several tens of ps, limited by the response time of the electric contour to control the applied voltage. ${ }^{15}$ As to the device operation temperature, at present, IXs are mainly explored at temperatures up to $100 \mathrm{~K}^{30}$ It is worth noting however that IXs were recently realized at room temperature in transition-metal dichalcogenide (TMD) heterostructures and the improvement of the TMD sample quality is in progress. ${ }^{31}$ We would like to add that using chiral metasurfaces ${ }^{19-21}$ instead of simple metal stripe gratings of the current work will allow to control the circular polarization of IX emission.

To conclude, we propose voltage tunable excitonic metasurface devices-integrated optoelectronic devices with voltage control of the polarization state of light, based on tunable emission of indirect excitons in coupled quantum well semiconductor heterostructure and plasmonic metasurface made of metal grating. Several modifications of such excitonic metasurface devices are considered, which allow us to tune the degree of linear polarization of emission in a broad range from linearly polarized along grating to perpendicular to grating, depending on the parameters of the gratings.
See supplementary material for 2D polar diagrams of far-field emission at resonant energy for the proposed light emitting devices with silver gratings and calculated characteristics (linear polarized components of the emission spectra and respective DLP) of the proposed light emitting devices with gold and titanium nitride gratings.

This work was supported by Russian Science Foundation (Grant No. 16-12-10538), NSF Grant No. 1640173 and NERC, a subsidiary of SRC, through the SRCNRI Center for Excitonic Devices. The authors are thankful to $\mathrm{T}$. Weiss for fruitful discussions.

${ }^{1}$ G. Lozano, S. R. K. Rodriguez, M. A. Verschuuren, and J. Rivas, Light Sci. Appl. 5, e16080 (2016).

${ }^{2}$ J. Hetterich, G. Bastian, N. A. Gippius, S. G. Tikhodeev, G. von Plessen, and U. Lemmer, IEEE J. Quantum Electron. 43, 855 (2007).

${ }^{3}$ N. C. Panoiu and R. M. J. Osgood, Opt. Lett. 32, 2825 (2007).

${ }^{4}$ R. A. Pala, J. White, E. Barnard, J. Liu, and M. L. Brongersma, Adv. Mater. 21, 3504 (2009).

${ }^{5}$ F. Neubrech, C. Huck, K. Weber, A. Pucci, and H. Giessen, Chem. Rev. 117, 5110 (2017).

${ }^{6}$ V. I. Belotelov, L. L. Doskolovich, and A. K. Zvezdin, Phys. Rev. Lett. 98, 077401 (2007).

${ }^{7}$ J. Y. Chin, T. Steinle, T. Wehlus, D. Dregely, T. Weiss, V. I. Belotelov, B. Stritzker, and H. Giessen, Nat. Commun. 4, 1599 (2013).

${ }^{8}$ D. Floess, T. Weiss, S. Tikhodeev, and H. Giessen, Phys. Rev. Lett. 117, 063901 (2016).

${ }^{9}$ N. Liu, T. Weiss, M. Mesch, L. Langguth, U. Eigenthaler, M. Hirscher, C. Sönnichsen, and H. Giessen, Nano Lett. 10, 1103 (2010).

${ }^{10}$ A. Tittl, H. Giessen, and N. Liu, Nanophotonics 3, 157 (2014).

${ }^{11}$ J. A. Jackman, A. R. Ferhan, and N.-J. Cho, Chem. Soc. Rev. 46, 3615 (2017).

${ }^{12}$ G. Li, S. Chen, N. Pholchai, B. Reineke, P. W. H. Wong, E. Y. B. Pun, K. W. Cheah, T. Zentgraf, and S. Zhang, Nat. Mater. 14, 607 (2015).

${ }^{13}$ P. Zeng, J. Cadusch, D. Chakraborty, T. A. Smith, A. Roberts, J. E. Sader, T. J. Davis, and D. E. Gómez, Nano Lett. 16, 2651 (2016).

${ }^{14}$ A. A. High, E. E. Novitskaya, L. V. Butov, M. Hanson, and A. C. Gossard, Science 321, 229 (2008).

${ }^{15}$ A. G. Winbow, A. T. Hammack, L. V. Butov, and A. C. Gossard, Nano Lett. 7, 1349 (2007).

${ }^{16}$ P. Andreakou, S. V. Poltavtsev, J. R. Leonard, E. V. Calman, M. Remeika, Y. Y. Kuznetsova, L. V. Butov, J. Wilkes, M. Hanson, and A. C. Gossard, Appl. Phys. Lett. 104, 091101 (2014).

${ }^{17}$ S. G. Tikhodeev, A. L. Yablonskii, E. A. Muljarov, N. A. Gippius, and T. Ishihara, Phys. Rev. B 66, 045102 (2002).

${ }^{18}$ S. V. Lobanov, T. Weiss, D. Dregely, H. Giessen, N. A. Gippius, and S. G. Tikhodeev, Phys. Rev. B 85, 155137 (2012).

${ }^{19}$ A. A. Maksimov, I. I. Tartakovskii, E. V. Filatov, S. V. Lobanov, N. A. Gippius, S. G. Tikhodeev, C. Schneider, M. Kamp, S. Maier, S. Höfling, and V. D. Kulakovskii, Phys. Rev. B 89, 045316 (2014).

${ }^{20}$ S. V. Lobanov, T. Weiss, N. A. Gippius, S. G. Tikhodeev, V. D. Kulakovskii, K. Konishi, and M. Kuwata-Gonokami, Opt. Lett. 40, 1528 (2015).

${ }^{21}$ S. V. Lobanov, S. G. Tikhodeev, N. A. Gippius, A. A. Maksimov, E. V. Filatov, I. I. Tartakovskii, V. D. Kulakovskii, T. Weiss, C. Schneider, J. Geßler, M. Kamp, and S. Höfling, Phys. Rev. B 92, 205309 (2015).

${ }^{22}$ P. B. Johnson and R. W. Christy, Phys. Rev. B 6, 4370 (1972).

${ }^{23}$ See http://www.sspectra.com/sopra.html for dielectric susceptibility of $\mathrm{Al}_{0.33} \mathrm{Ga}_{0.67} \mathrm{As}$.

${ }^{24}$ T. W. Ebbesen, H. J. Lezec, H. F. Ghaemi, T. Thio, and P. A. Wolff, Nature 391, 667 (1998).

${ }^{25}$ M. M. J. Treacy, Phys. Rev. B 66, 195105 (2002).

${ }^{26}$ F. J. García de Abajo, Rev. Mod. Phys. 79, 1267 (2007).

${ }^{27}$ F. J. Garcia-Vidal, L. Martin-Moreno, T. W. Ebbesen, and L. Kuipers, Rev. Mod. Phys. 82, 729 (2010).

${ }^{28}$ G. V. Naik, V. M. Shalaev, and A. Boltasseva, Adv. Mater. 25, 3264 (2013).

${ }^{29}$ V. Borshch, S. V. Shiyanovskii, and O. D. Lavrentovich, Phys. Rev. Lett. 111, 107802 (2013).

${ }^{30}$ G. Grosso, J. Graves, A. T. Hammack, A. A. High, L. V. Butov, M. Hanson, and A. C. Gossard, Nat. Photonics 3, 577 (2009).

${ }^{31}$ E. V. Calman, M. M. Fogler, L. V. Butov, S. Hu, A. Mishchenko, and A. K. Geim, e-print arXiv:1709.07043. 